\title{
Differentially expressed genes in autosomal dominant osteopetrosis type II osteoclasts reveal known and novel pathways for osteoclast biology
}

\author{
Amélie E Coudert ${ }^{1}$, Andrea Del Fattore ${ }^{2}$, Céline Baulard ${ }^{3}$, Robert Olaso ${ }^{3}$, Corinne Schiltz ${ }^{1}$, Corinne Collet ${ }^{1,4}$, \\ Anna Teti ${ }^{5}$ and Marie-Christine de Vernejoul ${ }^{1,6}$
}

Autosomal dominant osteopetrosis type II (ADO II) is a rare, heritable bone disorder characterized by a high bone mass and insufficient osteoclast activity. Mutations in the CLCN7 gene have been reported to cause ADO II. To gain novel insights into the pathways dysregulated in ADOII osteoclasts, we identified changes in gene expression in osteoclasts from patients with a heterozygous mutation of CLCN7. To do this, we carried out a transcriptomic study comparing gene expression in the osteoclasts of patients with ADO II and healthy donors. Our data show that, according to our selection criteria, 182 genes were differentially expressed in osteoclasts from patients and controls. From the 18 displaying the highest change in microarray, we confirmed differential expression for seven by qPCR. Although two of them have previously been found to be expressed in osteoclasts (ITGB5 and SERPINE2), the other five (CES1 (carboxyl esterase 1), UCHL1 (ubiquitin carboxy-terminal esterase L1, also known as ubiquitin thiolesterase), WARS (tryptophanyl-tRNA synthetase), GBP4 (guanylate-binding protein 4), and PRF1) are not yet known to have a role in this cell type. At the protein level, we confirmed elevated expression of ITGB5 and reduced expression of WARS, PRF1, and SERPINE2. Transfection of ClC-7 harboring the G215R mutation into osteoclasts resulted in an increased ITGB5 and reduced PRF1 expression of borderline significance. Finally, we observed that the ADO II patients presented a normal or increased serum level of bone formation markers, demonstrating a coupling between dysfunctional osteoclasts and osteoblasts. Sphingosine kinase 1 mRNA was expressed at the same level in ADO II and control osteoclasts. In conclusion, these data suggest that in addition to an acidification dysfunction caused by the CLCN7 mutation, a change in ITGB5, PRF1, WARS, and SERPINE2 expression could be part of the osteoclastic phenotype of ADO II.

Laboratory Investigation (2014) 94, 275-285; doi:10.1038/labinvest.2013.140; published online 16 December 2013

KEYWORDS: ADO II; integrin $\beta 5$; osteoclasts; SERPINE2; WARS

Osteopetrosis is a rare and heritable bone disorder characterized by a high bone mass caused by insufficient activity of osteoclasts. ${ }^{1,2}$ Two main clinical forms of osteopetrosis have been identified. Autosomal recessive osteopetrosis is often lethal in early childhood if untreated. Homozygous mutations in several genes have been identified as being responsible for the development of recessive osteopetrosis: mutations of CAII and TCIRG1 impair osteoclast acid secretion. ${ }^{1}$ Homozygous mutation in either CLCN7 or OSTM1, which have closely related functions, also induces osteopetrosis and the inability of osteoclasts to resorb bone. ${ }^{3,4}$ It was recently shown that mutations in either TNFSF11 or TNFRSF11A, which are responsible for normal osteoclast differentiation, induce osteoclast-poor osteopetrosis. ${ }^{5,6}$

Autosomal dominant osteopetrosis (ADO II), also known as Albers-Schönberg disease or marble bones, is seen in adults who present mainly bone-related symptoms. ${ }^{7}$ Recent studies of a series of patients with this disease indicate that serious clinical complications, such as fractures, delayed consolidation, and osteomyelitis, are frequent. ${ }^{8,9}$ These patients can also

\footnotetext{
${ }^{1}$ Institut National de la Santé et de la Recherche Médicale U606, Hôpital Lariboisière, Paris, France; ${ }^{2}$ Regenerative Medicine Unit, Ospedale Pediatrico Bambino Gesù, Rome, Italy; ${ }^{3} \mathrm{CEA}$ — Institut de Génomique, Centre National de Genotypage, Evry, France; ${ }^{4}$ Service de Biochimie, Hôpital Lariboisière, Paris, France; ${ }^{5}$ Department of Biotechnological and Applied Clinical Sciences, Università degli studi dell'Aquila, L'Aquila, Italy and ${ }^{6}$ INSERM U606, Os et articulations, Bâtiment Viggo Petersen, Secteur Viole, Fédération de Rhumatologie, Hôpital Lariboisière, Paris, France

Correspondence: Professor M-C de Vernejoul, MD, INSERM U606, Os et articulations, Bâtiment Viggo Petersen, Secteur Violet, 3ème étage, Fédération de Rhumatologie, Hôpital Lariboisière, 2 rue Ambroise Paré, 75010 Paris, France.
}

E-mail: christine.devernejoul@|rb.aphp.fr

Received 18 July 2013; revised 25 September 2013; accepted 22 October 2013 
present dental problems and osteoarthritis. In addition to orthopedic complications, retinal degeneration and marrow failure may be observed. ${ }^{8}$ Heterozygous mutations in the CLCN7 gene have been identified as being responsible for the ADO II. ${ }^{3,10}$ The severity of the disease is highly variable. This variability could be related to the presence of a modifying gene $^{11}$ or to polymorphism in the CLCN7 gene. ${ }^{12,13}$ Asymptomatic forms or healthy carriers are not exceptional. ${ }^{8,14}$ Patients have an increased number of osteoclasts; however, these are unable to form ruffled borders or to resorb bone efficiently. ${ }^{15}$

The CLCN7 gene encodes a chloride channel that is a member of the voltage-gated chloride channels family, 3,16 and has $\mathrm{Cl}^{-} / \mathrm{H}^{+}$exchanger properties. ${ }^{17}$ It resides mainly in the ruffled border of osteoclasts and in the late endosomes and lysosomes, where it resides with its $\beta$-subunit OSTM1. ${ }^{18}$ Mice deficient for the CLCN7 gene develop osteopetrosis in which the osteoclasts lack the ruffled border and the acidification ability and are unable to resorb bone. ${ }^{3}$ Lack of ClC-7 in the brain and kidney induces accumulation of lysosomal storage material. ${ }^{19}$

Around 50 heterozygous mutations of the CLCN7 gene have been described to cause ADO II..$^{8,10,13,20-22}$ The most common mutation is p.G215R ${ }^{23} \mathrm{ClC}-7$ is a homodimer, and it has been assumed that the mutated protein has a dominant-negative action. ${ }^{10,24}$ These heterozygous mutations may act on osteoclast function via the mislocalization of ClC-7, which is then unable to reach the ruffled membrane of the osteoclast. ${ }^{23} \mathrm{~A}$ defect in the acid microcompartment has sometimes, ${ }^{25}$ but not always, ${ }^{26,27}$ been observed. Finally, protein instability could also be induced by these mutations. ${ }^{24}$ Although several authors studied osteoclasts raised in culture from peripheral blood mononuclear cells (PBMCs) of ADO II patients, their results gave divergent data about the osteoclast phenotype of these patients, although decreased pit depth was always reported. ${ }^{25,27}$ Our objective was to identify changes in gene expression present in osteoclasts from patients with a heterozygous mutation of CLCN7. We therefore tried a different approach to investigate the osteoclasts of ADO II patients and conducted a transcriptomic study comparing gene expression in patients with ADO II and in healthy donors. Our aim was to get novel insights into pathways dysregulated in osteoclasts that might also be relevant for other forms of high bone mass disorders.

\section{MATERIALS AND METHODS Patients}

Patients with ADO II who were over 18 years of age and who were already part of our previous cohort ${ }^{9}$ were recruited for this study. All patients were systematically genotyped for CLCN7 mutations (NG_007567.1: RefSeqGene for exonic number and NM_001114331.2 for c.DNA). The sequencing analyses were performed with Life Technologies' reagents on an ABI 3130 sequencer using Sescape software version 4 (Life Technologies). Only 15 patients with a heterozygous mutation in the CLCN7 gene were selected for this study. In
14 out of the 15 patients, we evaluated serum tartrateresistant acid phosphatase $5 \mathrm{~b}$ (TRAP $5 \mathrm{~b}$ ) (IU/ml) by the IDS Immunodiagnostic System. We also measured serum type 1 collagen C-terminal telopeptide (CTx) and serum total type I procollagen $\mathrm{N}$-terminal propeptide (P1NP) using an ECLIA/ Cobas e601 Roche diagnostics kit. Bone formation was assessed in 14 patients by measuring bone alkaline phosphatase (BAP) using the Immunoenzymatique OSTASE ${ }^{\circledR}$ BAP kit provided by IDS. Bone mineral density (BMD) was measured at the femoral neck in eight patients using the same Lunar DPX-L (Lunar, Madison, WI, USA) densitometer. Sex- and age-adjusted values ( $Z$-score) were based on a French reference population between 20 and 89 years of age from several centers (provided by Lunar France).

We also recruited 31 unmatched healthy blood donors aged 20-61 years as controls for the osteoclast cultures. All research performed for this study was approved by the Ethics Committee of the Hôpital Lariboisière in Paris, France, and all individuals signed an informed consent document before entering the study.

\section{Osteoclast Cultures}

PBMCs were isolated from citrated blood samples from all the healthy donors and all the osteopetrotic patients by density-gradient centrifugation in Ficoll (Eurobio, France), washed in PBS (Life Technologies, France) and resuspended in $\alpha$-MEM (Life Technologies) containing $1 \%$ penicillin (Life Technologies), 1\% streptomycin (Life Technologies), 1\% L-glutamine (Life Technologies), and 10\% heat-inactivated FCS (Hyclone, Thermoscientific, France). They were seeded at a density of $2 \times 10^{6}$ cells per $\mathrm{ml}$ on one-well chambers/slides (Lab-Tek, Dutscher, France) for RNA and protein extractions, and on eight-well chamber/slides (Lab-Tek) for TRAP staining and immunofluorescence. Cells were cultured in a humid atmosphere with $5 \% \mathrm{CO}_{2}$. After incubating overnight, the cultures were supplemented with recombinant human M-CSF ( $25 \mathrm{ng} / \mathrm{ml}$; Preprotech, France) and recombinant human RANK-L (30 ng/ml; Preprotech). The PBMCs were cultured for 14 days, with the medium changed every 3-4 days. ${ }^{28}$ At the end of the culture, the cells were washed with PBS, fixed with $4 \%$ paraformaldehyde (PFA) (Sigma-Aldrich, France), and stained for TRAP activity. Their nuclei were counterstained with methyl green. Multinucleated (number of nuclei >3) TRAP-positive cells were considered to be differentiated osteoclasts, and were counted under a Zeiss microscope.

\section{Isolation of Total RNA}

For the quantitative real-time RT-PCR analysis, total RNA was extracted from the cells of all the osteoclast cultures at the end of the culture period using the RNeasy kit (Qiagen, France), which excludes genomic DNA. At least $10 \mu \mathrm{g}$ of RNA was harvested from each cell culture sample using this method. The total RNA yield (ng) was determined spectrophotometrically using the NanoDrop ND-100 (Labtech, 
Table 1 Primers used for Real Time PCR

\begin{tabular}{|c|c|c|c|c|}
\hline Gene & Forward primer & Reverse primer & Amplicon size & Efficacity (\%) \\
\hline CES1 & 5'-AAGACGGTGATAGGAGACCACG-3' & 5'-AGCAAAGTTGGCCCAGAATTT-3' & 129 & 99 \\
\hline ITGB3 & 5'-GCCCTGCTCATCTGGAAACTC & 5'-CCCGGTACGTGATATTGGTGA-3' & 151 & 99 \\
\hline WARS & 5'-TGGACGTGTCTITCATGTACCTG-3' & 5'-CATGGCTCCGCTGGTGTAAT-3' & 89 & 94 \\
\hline Serpine2 & 5'-GCTGAAAGTTCTTGGCATTACTGAC-3' & 5'-GCAAGATATGAGAAACATGGAGGTT & 101 & 94 \\
\hline Perforin 1 & 5'-AACTITGCAGCCCAGAAGACC & 5'-GGGAGTGTGTACCACATGGAAA-3' & 96 & 97 \\
\hline GBP4 & 5'-AACAGGTTGAGTGGGACTATAAGCTAGT-3' & 5'-GTGAGGGCTITGTCTGACTGC-3' & 127 & 87 \\
\hline OSBP & 5'-TGGAAAAGGAATCCTITACCGAA-3' & 5'-AGCATTGAGAGTCAGAGCAAGCT-3' & 75 & 97 \\
\hline TBP & $5^{\prime}$-CCCGAAACGCCGAATATAATCC-3' & 5'-GACTGTTCTTCACTCTTGGCTC-3' & 130 & 96 \\
\hline
\end{tabular}

France). Total RNA profiles were recorded using a Bioanalyzer 2100 (Agilent). RNA integrity numbers were determined, and the mean value found was $9.6 \pm 0.4$ s.d. The coefficient of variation (CV) was 4.4.

\section{Probe Synthesis, Hybridization, and Detection}

cRNA was synthesized, amplified, and purified using the Illumina TotalPrep RNA Amplification Kit (Ambion, France) following the manufacturer's instructions from the RNA extracted from all the osteoclast cultures. Briefly, $200 \mathrm{ng}$ of RNA was reverse transcribed. After second-strand synthesis, the cDNA was transcribed in vitro and cRNA labeled with biotin-16-UTP. Labeled probe hybridization to Illumina BeadChips human WG-6v2 was carried out using Ilumina's BeadChip WG-6v2 protocol. These beadchips contain 48701 unique 50-mer oligonucleotides in total, with hybridization to each probe assessed at 30 different beads on average. In all, 22403 probes $(46 \%)$ are targeted at ReferenceSequence $\left(\right.$ RefSeq) ${ }^{29}$ transcripts and the remaining 26298 (54\%) are for other transcripts, generally less well characterized (including predicted transcripts).

Beadchips were scanned on the Illumina beadArray 500GX Reader using Illumina BeadScan image data acquisition software (version 2.3.0.13). Illumina BeadStudio software (version 1.5.0.34) was used for preliminary data analysis. Several quality control procedures were used to assess the quality metrics of each run. Total RNA control samples were analyzed with each run. The Illumina BeadStudio software was used to view the control summary reports, scatter plots of the total RNA control results from different days, and scatter plots of daily run samples. The scatter plots compared control vs control or sample vs sample, and calculated a correlation coefficient. Viewing the scatter plots determined whether controls from different days varied in quality, which would indicate a reduction in assay performance, and highlighted any samples that were of lower quality. The control summary report was generated by BeadStudio software, which evaluates the performance of the built-in controls of the BeadChips across particular runs. This allows the user to look for variation in signal intensity, hybridization signal, background signal, and the background/noise ratio for all the samples analyzed in that run. Data are expressed as $\log 2$ ratios of fluorescence intensities of the experimental samples and the common reference sample. The Illumina data were then normalized using the 'normalize quantiles' function in the BeadStudio Software.

\section{Real-Time PCR}

Real-time PCR assays were carried out following MIQE guidelines. ${ }^{30}$ Briefly, total RNA was reverse transcribed using Superscript III and oligo(dT) primers (Life Technologies) according to the manufacturer's instructions. Real-time quantitative PCR was carried out using the SYBR-green master mix (Applied BioSystems, France) in an Mx $3005 \mathrm{P}$ Thermocycler (Agilent). PCR conditions were $95^{\circ} \mathrm{C}$ for $10 \mathrm{~min}$ followed by 40 cycles of $95^{\circ} \mathrm{C}$ for $15 \mathrm{~s}$ and $60{ }^{\circ} \mathrm{C}$ for $1 \mathrm{~min}$. At the end of the amplification reaction, melting-curve analyses were performed to confirm the specificity and the integrity of the PCR products by the presence of a single peak. Gene-specific primers were designed inside or close to the microarray sequence targeted, using Primer Express software (PE Applied Biosystems). Primer sequences of all the genes analyzed are shown in Table 1. The absence of cross-contamination and primer dimers was checked on genomic DNA and water. Standard curves were generated from assays made with serial dilutions of reference cDNA to calculate PCR efficiencies $\left(100 \pm 15 \%\right.$, with $\left.r^{2} \geq 0.996\right)$. Cq samples were transformed into quantity values using the formula $(1 \pm \text { Efficiency })^{-\mathrm{Cq}}$. Only means of triplicates with a $\mathrm{CV}$ of $<10 \%$ were analyzed. Interplate variation was below $10 \%$. Values were normalized to the geometric mean of the two normalization factors (NF) found to be the most stable in all the samples using the geNorm approach (out of eight $\mathrm{NF}$ chosen on the basis of microarray data and tested by 
qPCR). ${ }^{31}$ The two NFs used were oxysterol-binding protein (OSBP) and TATA box-binding protein (TBP), which displayed a pairwise variation $V 2 / 3=0.077$.

\section{Western Blotting}

Cell extracts were prepared using lysis buffer $(\mathrm{NaCl} 150 \mathrm{mM}$, Tris-HCl $200 \mathrm{mM}$, Triton X-100 0.5\%, deoxycholate $0.5 \%$, sodium orthovanadate $1 \mathrm{mM}$ ) containing protease inhibitors (Roche Applied Science, France). Protein samples were diluted in $2 \times$ Laemmli SDS loading buffer (Sigma Aldrich), and heated at $95^{\circ} \mathrm{C}$ for $5 \mathrm{~min}$. A quantity of $15 \mu \mathrm{g}$ of proteins (determined by using ADVIAr1650; Bayer Diagnostics, France) was resolved on an $8 \%$ acrylamide gel, and then transferred onto a polyvinylidene difluoride-Hybond-P membrane (Hybond-P; GE Healthcare, France). Blots were saturated with blocking buffer (Sigma Aldrich) and then probed with primary antibodies against CES1 (carboxyl esterase 1) (1/500), ITGB5 (1/500), PRF1 (1/1000), SERPINE2 (1/200), WARS (tryptophanyl-tRNA synthetase) (1/500), GBP4 (guanylate-binding protein 4) (1/500), UCHL1 (ubiquitin carboxy-terminal esterase L1, also known as ubiquitin thiolesterase) (1/200) and GAPDH (1/200) (all antibodies from Abcam, UK). After incubating overnight at $4{ }^{\circ} \mathrm{C}$, the membranes were washed two times with TBS $0.1 \%$ Tween-20, and $0.5 \%$ blocking buffer. They were then incubated for $1 \mathrm{~h}$ at room temperature with horseradish peroxidase-conjugated secondary antibodies. Following incubation with the appropriate secondary antibodies (from Santa Cruz) (used at a 1/10000 dilution), the membranes were washed and the signals were visualized with the West pico ECL system (Thermoscientific, France) on a Fujifilm Intelligent Dark Box LAS 3000 lite (version 2.2). The intensity of the band was quantified using Image Gauge software (version 4.2), and then expressed as a ratio of the protein band intensity on the GADPH band intensity.

\section{Immunocytofluorescence}

At the end of the culture, the cells were washed in PBS $1 \times$, and then fixed for $20 \mathrm{~min}$ at room temperature with PFA 4\%. The fixed cells were washed several times in PBS $1 \times$, and then incubated in $\mathrm{PBS} / 3 \% \mathrm{BSA} / 0.001 \%$ Tween for $1 \mathrm{~h}$ at room temperature. The primary monoclonal antibodies (against ITGB3, ITGB5, CES 1, PERFORIN 1, SERPINE2, WARS, GBP4, and UCHL1; all from Abcam; 1/100) were incubated on cells overnight at $4{ }^{\circ} \mathrm{C}$. The cells were then washed several times in PBS $1 \times$ and incubated for $1 \mathrm{~h}$ at room temperature with the Alexa488-conjugated secondary antibodies (Molecular Probes, France and Jackson ImmunoResearch, UK). The cells were finally incubated with Texas Red ${ }^{\mathbb{R}} \mathrm{X}$-phalloidin (Life Technologies) for $20 \mathrm{~min}$ and the nuclei counterstained with DAPI. Slides were analyzed by using a Zeiss Axioplan.

\section{Human Osteoclast Transfection}

Human osteoclasts were isolated from healthy donors and then cultured as described previously. After reaching $80 \%$ confluence, the cells were transfected according to the Amaxa protocol and as described previously. ${ }^{32}$ Briefly, after cell harvesting, $1 \times 10^{6}$ cells were pelleted and resuspended in human monocyte nucleofector solution (Lonza, Italy) to which $2 \mu \mathrm{g}$ of plasmid were added. The transfected vectors were either empty or overexpressed the WT CLCN7 or the G215R CLCN7 mutation. The solution was added to Amaxa electrode cuvette and electroporated in an Amaxa Nucleofector II using program Y-010. Immediately afterwards, the cells were resuspended in the culture medium containing MCSF and RANK-L, and cultured for $48 \mathrm{~h}$ in this osteoclast-differentiating medium. The cells were then harvested and the total RNA extracted, then retrotranscribed, and finally subjected to a $\mathrm{qPCR}$ for the expression of CLCN7 and the candidate genes. The transfection was considered to be efficient when the CLCN7 gene expression showed at least a twofold increase compared with the empty vector transfection.

\section{Statistical Analysis}

Data are presented as means \pm 2 s.e.m. Statistical comparison were made using Student's $t$-test or Mann-Whitney when appropriate, with $P<0.05$ being considered significant.

\section{RESULTS}

\section{The Patients Exhibit a Typical ADO II Phenotype}

The ADO II group was composed of 15 patients, 6 men and 9 women, and the control group consisted of 31 healthy blood donors, 18 men and 13 women. Eleven ADO II patients belonged to 4 different families and the other 4 patients were unrelated. The ages ranged from 18 to 84 years (mean age $49 \pm 10$ years) in the ADO II patient group, and from 20 to 61 years old (mean age $38 \pm 5$ years) in the healthy donor group. The ADO II patients had a total of six different CLCN7 mutations, half of them localized in the C-terminal domain of the protein (Figure 1a). ${ }^{33}$ The most frequent mutations responsible for $\mathrm{ADO} \mathrm{II}^{10,23}$ were also represented in our patient group: five related patients had the p.G215R mutation and three unrelated patients the p.R767W mutation. All the patients had a higher than normal (2-5 U/ml) serum TRAP level, with values ranging from 5.3 to $60.9 \mathrm{U} / \mathrm{ml}$ (mean value $33.9 \pm 9.3 \mathrm{U} / \mathrm{ml}$ ) (Figure $1 \mathrm{~b}$ ). The bone resorption marker serum CTx (normal range from 100 to $700 \mathrm{pg} / \mathrm{ml}$ ) was within the normal range for all but two of the osteopetrotic patients, with an overall mean of $445 \pm 261 \mathrm{pg} / \mathrm{ml}$. The mean ratio of CTx/TRAP was $24 \pm 18$ $(\mathrm{pg} / \mathrm{U})$ with a ratio ranging from 2 to 106 . This ratio was markedly decreased compared with the normal range $(50-140 \mathrm{pg} / \mathrm{U})$ for all the patients but two. The serum BAP level (normal range from 5 to $15 \mathrm{ng} / \mathrm{ml}$ ) was elevated in 6 of the 14 patients, ranging from 17 to $64 \mathrm{ng} / \mathrm{ml}$. The other eight patients presented a normal BAP level, ranging from 6.9 to $13.2 \mathrm{ng} / \mathrm{ml}$. The overall mean value of the BAP level in the ADO II patient group was $21.3 \pm 9.6 \mathrm{ng} / \mathrm{ml}$, higher than the normal range (Figure 1b). The serum BAP level was not 
a
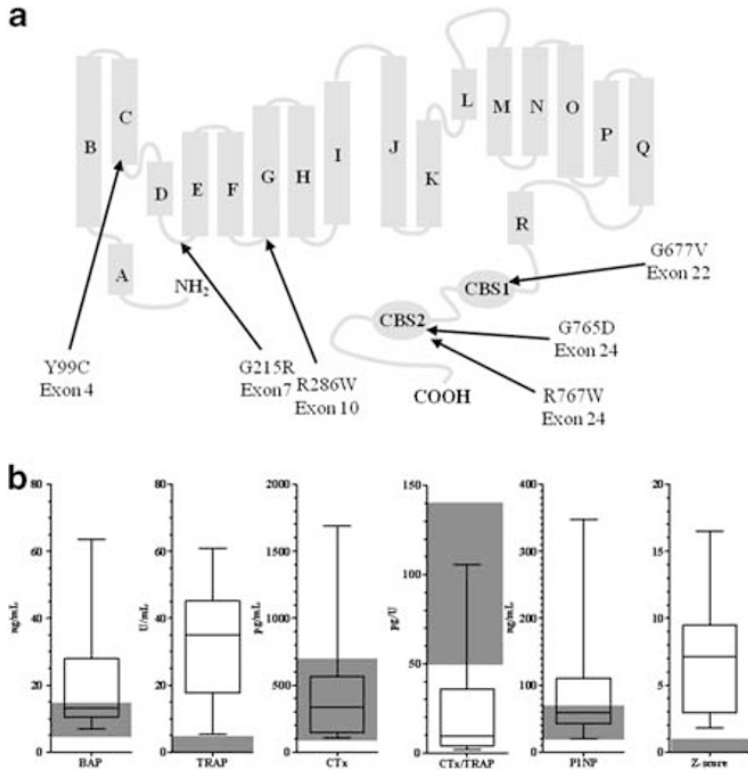
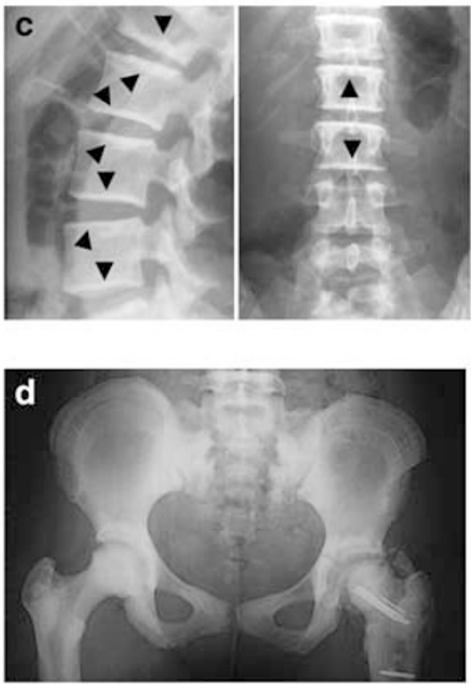

Figure 1 Phenotype and CLCN7 mutations of autosomal dominant osteopetrosis type II (ADO II) patients. (a) Localization of the different CLCN7 mutations presented by the patients (adapted from Dutzler et $a^{67}$ ). The patients presented six different CLCN7 mutations variously positioned on the CLC7 protein, with three of them localized in the intracellular C-terminal region, and the others in the transmembrane region. The most common mutation, p.G215R, was present in five of our osteopetrotic patients. (b) The levels for serum bone markers such as bone alkaline phosphatase (BAP), tartrate-resistant acid phosphatase (TRAP), serum type 1 collagen C-terminal telopeptide (CTx), the CTx/TRAP ratio, and serum total type I procollagen $\mathrm{N}$-terminal propeptide (P1NP), and the femoral BMD Z-score of 14 out of 15 osteopetrotic patients are shown here. The gray zone corresponds to the normal range. (c and d) Typical X-ray features of osteopetrotic patients. (c) Dense bands of sclerosis parallel to the vertebral endplates ('sandwich vertebrae', arrowheads) and (d) concentric arcs of sclerosis within the iliac wings ('bone within bone').

correlated with their serum TRAP level. Analysis of the P1NP (normal range from 10 to $70 \mathrm{ng} / \mathrm{ml}$ ) revealed a mean higher than the range of the normal values at $89.5 \pm 50.0 \mathrm{ng} / \mathrm{ml}$. This level was elevated in six of the osteopetrotic patients. According to the previously defined osteopetrotic score, ${ }^{34}$ the patients presented a mild ADO II phenotype and the most severe clinical case (score 2) had sustained more than 10 fractures, but none of the patients had visual impairment or bone marrow failure. However, the femur neck Z-score (when assessed) was increased as expected for osteopetrotic patients $(+7.3 \pm 3.4)$ (Figure 1b), and was not correlated to any formation or resorption marker. All the patients had the typical X-ray features of ADO II, with sandwich vertebrae and bone in bone features (Figures $1 \mathrm{c}$ and $\mathrm{d}$ ).

\section{qPCR Validation of Transcriptome Analysis of Osteoclast Cultures Reveal Seven Genes Differentially Expressed in ADO II Patients and Healthy Donors}

From each of the 15 ADO II patients and the 31 healthy blood donors, PBMCs were purified and cultured for 14 days with MCS-F and RANK-L in order to differentiate them in osteoclasts. To assess the homogeneity of our cultures, a TRAP staining was carried out and osteoclasts were counted. We show that morphologically our cultures were comparable (Figure 2a), and that the number of differentiated osteoclasts after 14 days was not different in the cultures from the ADO II patients and from the healthy donors (ADO II:
$50.49 \pm 21.41$ OC per $\mathrm{mm}^{2}$; healthy donors: $39.75 \pm 9.19$ OC per $\mathrm{mm}^{2}$; Figure $2 \mathrm{~b}$ ). We next analyzed the expression of the OC marker genes by qPCR, and showed that there was no difference between the OC cultures from the ADO II patients and the healthy controls with regard to the expression of TRAP, CTSK, or RANK (Figure 2c). We also showed that although the CLCN7 gene is mutated in the ADO II patients, the expression of this gene was not different in the two types of osteoclast cultures (Figure 2d).

As our two types of osteoclast cultures were comparable, RNAs were extracted and a transcriptomic analysis was carried out to look for differences at the transcription level. The stringency of our transcriptome analysis was a $P$-value $<0.05$ without any correction and 183 gene were differentially expressed (Supplementary Table 1). We selected 18 candidate genes showing the greatest difference in expression between the osteopetrotic and the control osteoclasts (from a 13\% increase and from a 20\% decrease of expression between patients and healthy donor osteoclasts). Out of these 18 selected genes, we were able to confirm differential expression of only seven of them by qPCR; these are shown in Figure 2e (transcriptome and qPCR data). The qPCR differences were as follows: ITGB5 $(+53 \%$ in the osteopetrotic patient $v \mathrm{~s}$ healthy donor control; $P=0.005), C E S 1(+323 \%$ osteopetrotic patient $v s$ healthy donor control; $P=0.003), P R F 1$ $(-32 \%$ osteopetrotic patient $v s$ healthy donor control; $P=0.044)$, SERPINE2 $(-53 \%$ osteopetrotic patient $v s$ 
a

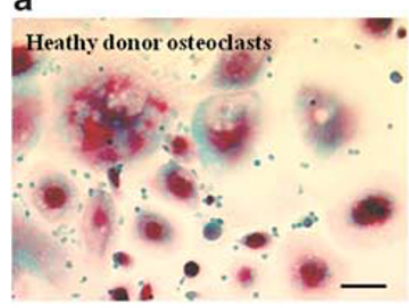

C

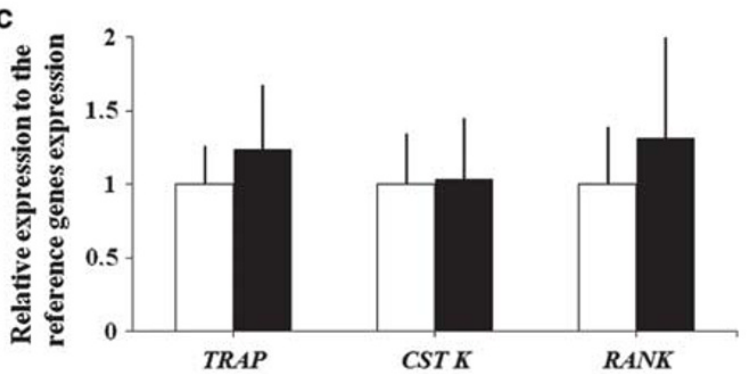

e
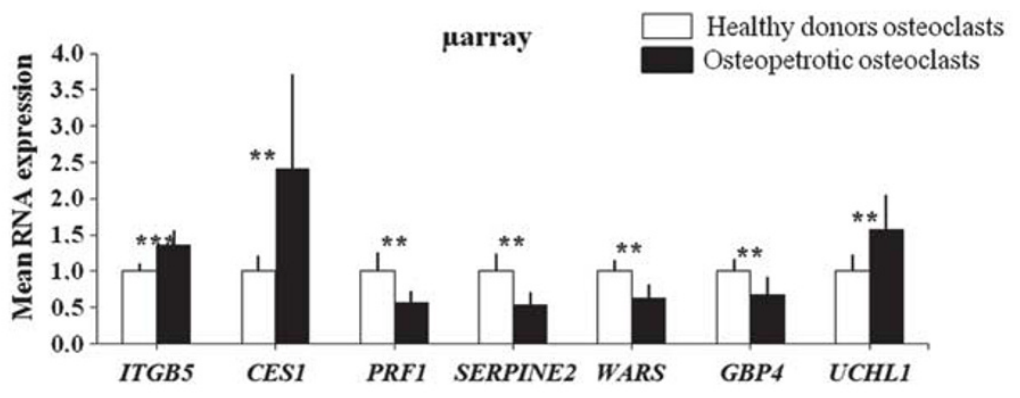

f

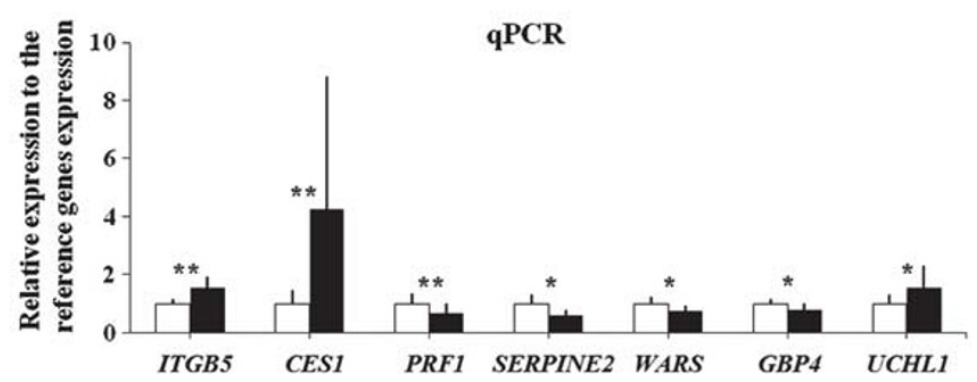

Figure 2 Quantitative polymerase chain reaction ( $\mathrm{PPCR}$ ) validation of the transcriptome analysis of osteoclast cultures reveals seven genes differentially expressed in autosomal dominant osteopetrosis type II (ADO II) patients and healthy donors. (a) Tartrate-resistant acid phosphatase (TRAP) staining of 14-day cultures of osteoclasts from a healthy donor (left) and an osteopetrotic patient (right). No difference could be observed at the morphological level. The pictures shown here are representative of the observations made on the osteoclast cultures from 15 osteopetrotic patients and 31 healthy donors (scale bar: $50 \mu \mathrm{m}$ ). (b) The number of multinucleated and TRAP-positive osteoclasts was evaluated for all the control and osteopetrotic osteoclast cultures. No significant difference was observed between the two types of cultures. (c) The expression levels of three osteoclastic differentiation markers were evaluated by real-time quantitative polymerase chain reaction (RT-qPCR) from all the osteopetrotic and healthy donor osteoclast cultures. No significant difference was observed between the two types of culture for the expression of TRAP, CTSK, or RANK. (d) CLCN7 expression (on the microarray) was not different in the osteopetrotic osteoclasts when compared with the healthy donor osteoclasts, even though the osteopetrotic osteoclasts carried a CLCN7 mutation. (e) Significant differences in the expression of ITGB5, CES1, PRF1, SERPINE2, WARS, GBP4 and UCHL1 in osteoclasts from healthy donors and osteopetrotic patients obtained by microarray. (f) Significant differences in the expression of ITGB5, CES1, PRF1, SERPINE2, WARS, GBP4 and UCHL1 in osteoclasts from healthy donors and osteopetrotic patients validated by qPCR. Healthy donors in white bars, $n=31$; osteopetrotic patients in black bars, $n=15$. ${ }^{*} P<0.05,{ }^{* *} P<0.005$, and ${ }^{* *} P<0.001$.

healthy donor control; $P=0.03)$, WARS ( $-34 \%$ osteopetrotic patient $v s$ healthy donor control; $P=0.023)$, GBP4 $(-31 \%$ osteopetrotic patient $v s$ healthy donor control;
$P=0.013)$, and UCHL1 $(+68 \%$ osteopetrotic patient $v s$ healthy donor control; $P=0.031$ ) (Figure $3 \mathrm{~g}$ ). Our results validated these genes as candidate ADO II biological markers. 
a

$\beta 5$
Serpine2

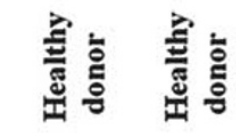

हैํㄹㄹ

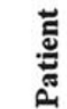

CES1

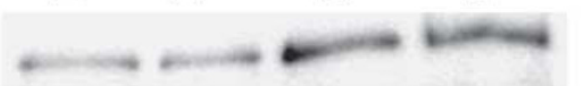

1.16

3.65

4.52

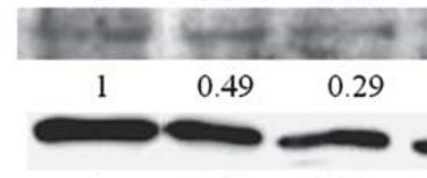

1

0.83

0.87

0.87

\section{GAPDH}

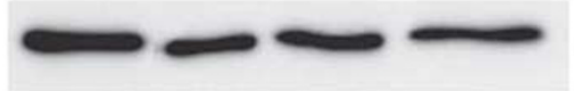

b

b
WARS
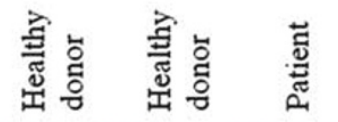

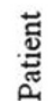

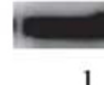

1

\begin{abstract}
0.50
\end{abstract}
0.10

0.04

Perforin 1

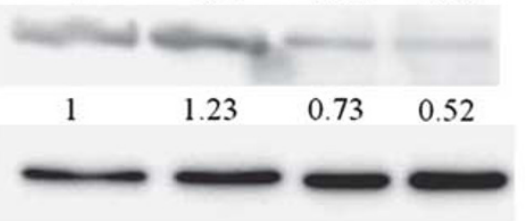

\section{GAPDH}

$95 \mathrm{KDa}$ c

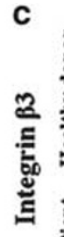

DAPI
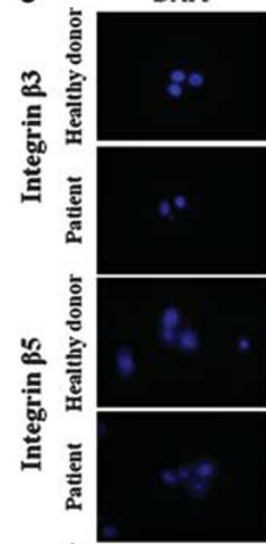

$33 \mathrm{KDa}$

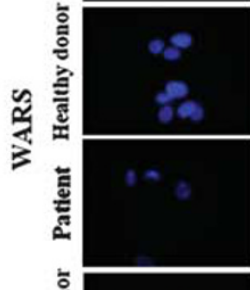

$53 \mathrm{KDa}$

$66 \mathrm{KDa}$

$33 \mathrm{KDa}$
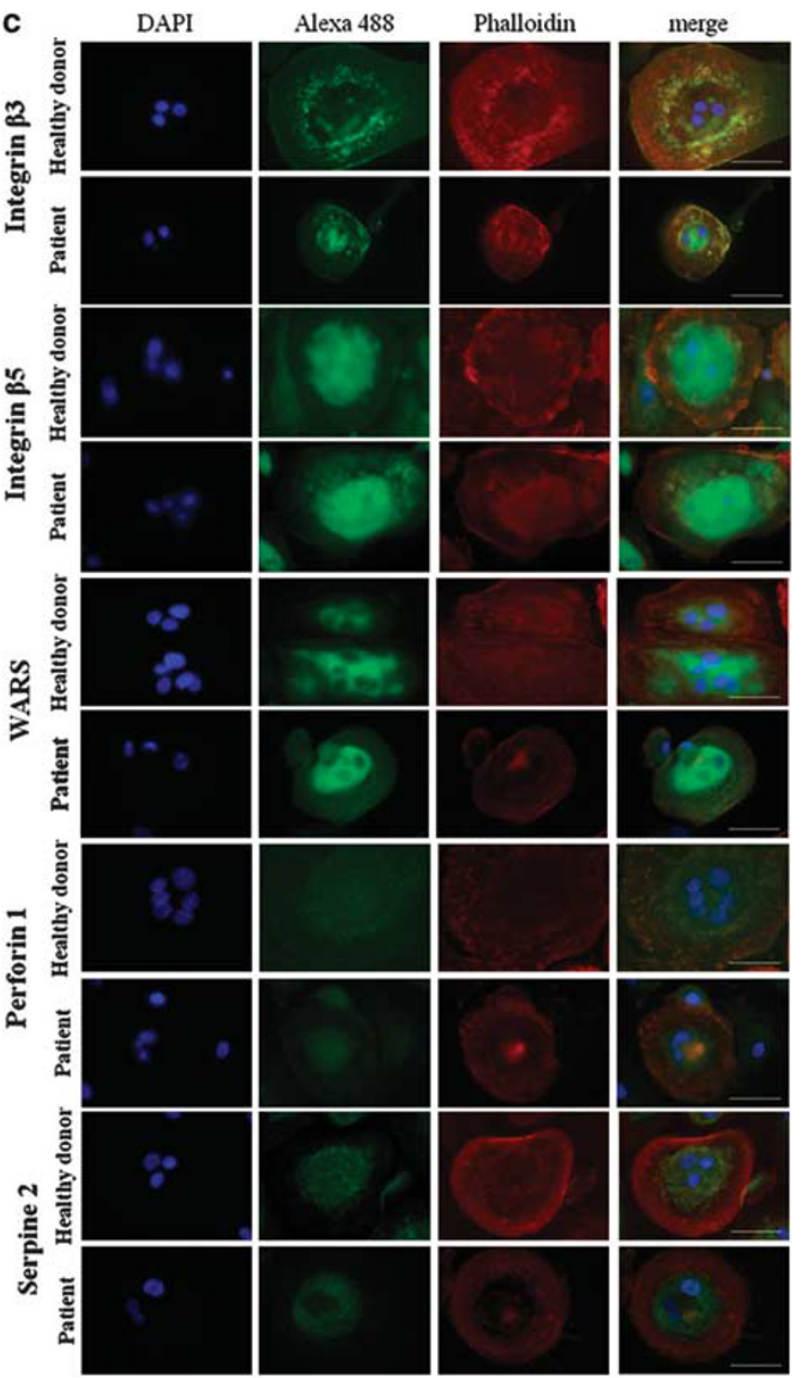

Figure 3 Increase in integrin $\beta 5$ and decreases in SERPINE2, Perforin 1, and WARS are validated at the protein level in osteoclasts from osteopetrotic patients vs those from healthy donors. (a) Western blot of proteins extracted from two osteopetrotic patients (mutations G215R and R767W) and two healthy donor osteoclast cultures showing greater expression of the ITGB5, no difference in CES1 expression, and lower expression of SERPINE2 in osteopetrotic osteoclasts vs healthy donor osteoclasts. The expression is expressed as the ratio of the protein expression over GAPDH expression. (b) Western blot of proteins extracted from two osteopetrotic patients and two healthy donor osteoclast cultures showing lower expression of WARS and PRF1. The expression is expressed as the ratio of the protein expression over GAPDH expression. (c) Immunofluorescence was performed on all the osteopetrotic and healthy donors osteoclasts. The immunostainings shown here are representative of those observed for all the osteoclast cultures performed. It shows the cytoplasmic expression of ITGB3, ITGB5, SERPINE2, PRF1, and WARS (revealed by a secondary antibody coupled with Alexa488) (first column) in human osteoclasts in comparison with the phalloidin localization (Texas Red) (second column), nuclei stained with 4',6-diamidino-2-phenylindole (DAPI) (third column), and a merge image (fourth column) (scale bar: $50 \mu \mathrm{m}$ ).

ITGB3 mRNA expression was not significantly different in either the microarray or qPCR data $(+13 \%$ osteopetrotic patient $v s$ healthy donor control; $P=0.2509$ ). We also analyzed the SPHK1 mRNA expression. Although its expression was significantly higher in ADO II patients in the microarray $(+109 \%$ osteopetrotic patient vs healthy donor control; $P=0.028$ ), no significant difference was observed by qPCR $(+3 \%$ osteopetrotic patient $v s$ healthy donor control; $P=0.86$ ). We also carried out a DAVID analysis of our transcriptome results. ${ }^{35,36}$ This clustering analysis reveals only an enrichment statistically significant for the organelle lumen and the membrane-enclosed lumen clusters including the same 33 genes (for details, see Supplementary Tables 2 and 3 ).

\section{Changes at the Protein Level Between ADO II and Healthy Donor Osteoclasts were Observed for Four Markers: ITGB5, WARS, SERPINE2, and PRF1}

To validate the changes in expression observed by qPCR, we assessed the protein expression of these seven candidate genes by western blotting of the proteins extracted from osteopetrotic and healthy donor osteoclasts. We confirmed 
the increased expression of ITGB5 (278\%) and the decreased expression of SERPINE2 (51\%), WARS (68\%), and PRF1 (49\%) in osteopetrotic osteoclasts compared with the healthy donor osteoclasts (Figures $3 \mathrm{a}$ and $\mathrm{b}$ ). No difference in CES1 expression was observed. We were not able to detect UCHL1 and GBP4 by western blotting. We then conducted immunofluorescence on osteopetrotic and healthy donor osteoclasts to investigate the presence of the proteins coded by the candidate genes in human osteoclasts, and the possible different intracellular localization of the proteins we had detected by western blot. As a control, we stained the osteoclasts for ITGB3 and observed that, as expected, it colocalized with phalloidin in both ADO II and healthy-donor osteoclasts. In contrast, ITGB5, WARS, SERPINE2, CES1, and PRF1 were present in the cytoplasm of both ADO II and healthy donor osteoclasts, but did not colocalize with phalloidin (Figure 3c). Immunostainings did not allow us to evidence any decrease in the protein expression or any change of localization of the protein of interest between the ADO II and healthy donor osteoclasts.

\section{Gene Expression Modifications in the Transfection Model}

To validate the differential expression of our candidate genes in osteoclasts induced by CLCN7 mutations, we decided to transfect a mutated CLCN7 plasmid into primary osteoclasts from healthy blood donors as described previously. ${ }^{32} \mathrm{We}$ carried out four successful transfections and compared gene expression in the osteoclasts transfected with the mutated CLCN7 plasmid to the ones transfected with the WT CLCN7 plasmid. We observed, as expected, that the CLCN7 expression in osteoclasts transfected with WT CLCN7 vector was increased in comparison with those transfected with the empty vector (Figure 4a). We then analyzed the expression of our four genes of interest. We were able to detect a trend toward the increased expression of ITGB5 $(P<0.08)$ and decreased expression of PRF1 $(P<0.06)$, and no significant change in WARS and SERPINE2 in (Figure 4b). These results confirmed the impact of the CLCN7 mutation on the transcription regulation of the ITGB5 and PERF1.

\section{DISCUSSION}

In this study, we observed that several genes were differentially expressed in osteoclasts raised in culture from PBMC taken from ADO II patients and healthy blood donors. Among these genes, we validated the ITGB5, WARS, SERPINE2, and PERF1 in our different assays. We found that change in expression of ITGB5 and PERF1 were indeed observed not only at the gene and protein levels but was also triggered in human osteoclasts by transfection of a mutated CLCN7 plasmid.

Gene expression profiling is a powerful tool for investigating the changes induced by mutation in human samples. In osteoclasts, it has previously been used to investigate changes occurring in Paget's disease of bone. ${ }^{37,38}$ In our study, osteoclast cultures were similar in patients and donors in terms of cell number and differentiation, which enabled us to compare gene expression in osteoclasts of both provenances. We observed 183 genes differentially expressed in ADO II osteoclasts. Of this group of genes, the 18 most highly regulated candidates were further analyzed, which comprised several genes not previously described in osteoclasts. For only seven genes, differential expression was confirmed qPCR. Among these were the ubiquitously expressed genes GBP4 and UCHL1, $, 39,40$ and also the gene CES1 previously reported to be expressed in macrophages. ${ }^{41}$ Although we were able to confirm that their RNA were expressed in human osteoclasts, we were not able to confirm any change at the protein expression level.

SERPIN are part of the plasminogen system involved in matrix remodeling and thrombus formation. SERPINE2, also known as protease nexin-1, PN-1, belongs to the serine protease inhibitor superfamily. ${ }^{42}$ SERPINE2 is expressed in a wide variety of tissues. It was identified as a potential candidate susceptibility gene for the chronic obstructive pulmonary disease. ${ }^{43}$ SERPINE2 strongly influences fibrinolysis and thrombolysis ${ }^{44}$ SERPINE2 expression in murine osteoclasts was first demonstrated by Yang et al. ${ }^{45}$ It has been suggested that it may be involved in the removal of non-collagenous proteins that are present in the nonmineralized bone matrix, but are not required for osteoclast differentiation or for the resorption of the mineralized matrix. ${ }^{46}$ SERPINE2 was markedly decreased in ADO II osteoclasts, and we can speculate that it could be a compensatory mechanism for the decrease in the resorption function of ADO II osteoclasts.

Perforin 1 is a $66 \mathrm{kDa}$ pore-forming protein that polymerizes and forms a transmembrane, pore-like structure in the plasma membrane lipid bilayers ${ }^{47,48}$ of target cells. ${ }^{49}$ This channel is dependent on $\mathrm{Ca}^{2}{ }^{+}$, and delivers granzymes into the cytosol of target cells. This is the only currently known granzyme delivery molecule. ${ }^{47,48}$ Perforin 1 is expressed in cytotoxic natural killer lymphocytes. ${ }^{50}$ Perforin1-deficient mice are viable and have severe defect in natural killer cell-mediated cytolysis. ${ }^{51}$ PRF1 gene defects are responsible for familial hemophagocytic lymphohistiocytosis, a severe condition with immune deregulation. ${ }^{52}$ No bone phenotype has been reported for patients or mice deficient for PRF1. Demonstration of PRF1 expression in osteoclasts raises the question of whether its expression may not be restricted to cytotoxic immune cells and its decreased expression in osteopetrotic osteoclasts might deserve further study.

WARS expression was markedly decreased at both the gene and protein levels in ADO II osteoclasts. WARS catalyzes the aminoacylation of tRNA(Trp) with tryptophan to make it available to the cell's protein synthesis machinery. ${ }^{53}$ We had observed previously that osteoclasts can synthesize serotonin from tryptophan, and this triggers osteoclast differentiation as they express tryptophan hydroxylase $1 .{ }^{54}$ It is therefore possible that WARS is related to the osteoclast biology, but 
a

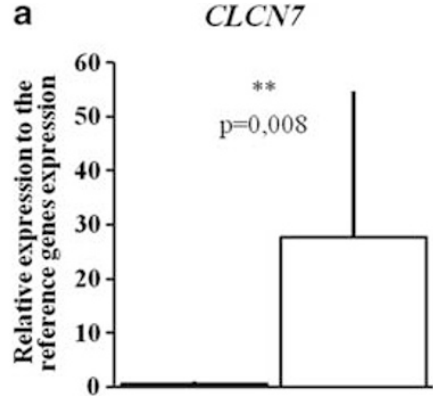

Empty $C L C N 7$ vector

WT CLCN7 vector b

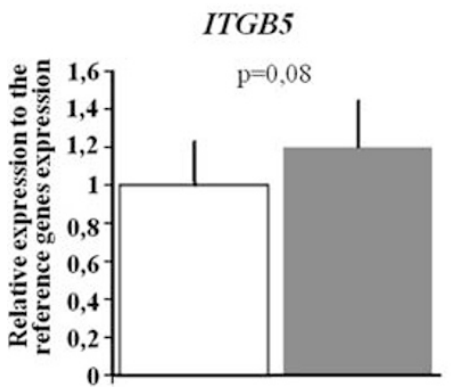

WARS

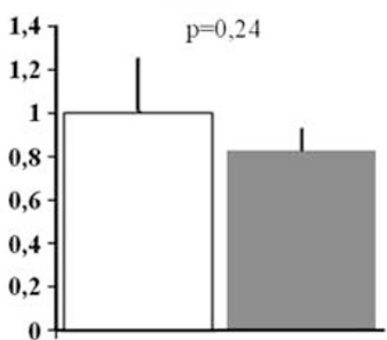

SERPINE2

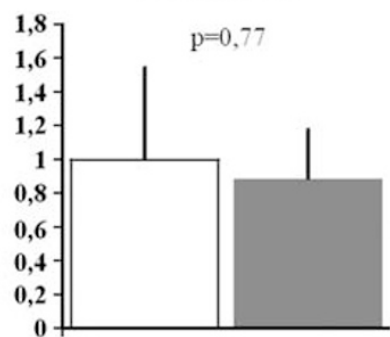

WT $C L C N 7$ vector G215R CLCN7 vector

PRF 1

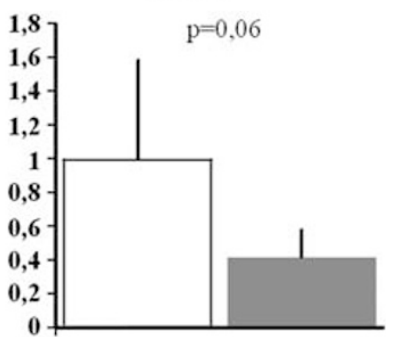

Figure 4 Human osteoclast transfection. Human osteoclasts from healthy blood donors were transfected with an empty, a wild-type (WT) or a G215Rmutated CLCN7 expression plasmid. (a) CLCN7 expression is significantly increased in the human osteoclasts transfected with the WT CLCN7 vector (white bar) in comparison with those transfected with the empty vector (hatched bar). (b) The expression of ITGB5, WARS, SERPINE2, and PRF1 was assessed by quantitative polymerase chain reaction (qPCR). No significant difference was observed for WARS and SERPINE2, but there was a trend for an increased expression of ITGB5 and a decreased expression of PRF1. This figure shows the mean of four independent successful transfection experiments, as assessed by the overexpression of CLCN7. Human osteoclasts transfected with the WT CLCN7 vector are represented by white bars; human osteoclasts transfected with G215R CLCN7 vector by gray bars. $P$-value from student test is reported above the bars.

we could not confirm it in the transfection experiment. WARS had not previously been observed in osteoclasts, and its function in this cell is unknown. Interestingly, the short form of WARS is inducible by interferon..$^{55}$ Furthermore, SERPINE2 and PRF1 are also indirectly or directly regulated by interferon. Kasper et $a l^{56}$ performed genome-wide gene expression profiling in the hippocampus of $\mathrm{Clcn} 7-/-$ mice. They observed in this tissue higher expression of several interferon-inducible genes pointing to the same pathway as in osteoclasts but in an opposite direction.

Among these candidate genes, ITGB5 was the most coherent target as it was overexpressed at the RNA and protein level and there was a trend toward increased levels after expression of mutated CLCN7 in human osteoclasts. ITGB $\beta 5$ is known to be more highly expressed in immature osteoclasts than in fully differentiated osteoclasts. ${ }^{57}$ Furthermore, $\alpha_{\mathrm{V}} \beta 5$ cannot replace $\alpha_{\mathrm{V}} \beta 3$ in osteoclastogenesis when the latter is missing. ${ }^{58}$ Finally, a lack of expression of integrin $\beta 5$ in female mice induces increased osteoclast function. ${ }^{59}$ In our ADO II osteoclasts, as reported previously, ${ }^{27}$ osteoclast differentiation was unchanged, at least when comparing the number of multinucleated, TRAP-positive cells and the expression of osteoclast-specific genes, such as CTSK, TRAP, and RANK. In addition, a morphological change in the motile structure of ADO II osteoclasts has been reported previously. ${ }^{27}$ Another group has reported that osteoclast motility was modified in ADO II patients with CLCN7 mutations. ${ }^{26}$ Finally, a single osteopetrotic patient with an unknown mutation presented changes in osteoclast integrins, including increased expression of integrin $\beta 5 .^{60}$ The cytoskeletal organization and mobility of osteoclasts are both dependent on integrin $\beta 3$ and its downstream signaling pathway involving Rho GTPase and vav3. ${ }^{61}$ We did not observe any change in integrin $\beta 3$ in ADO II osteoclasts, but it is possible that integrin $\beta 5$ overexpression could interfere with the $\beta 3$ intracellular signaling. Convergent morphological data point to a change in osteoclast motility in ADO II and our finding of higher level of integrin $\beta 5$ fits in with these observations.

Lysosomal function in osteoclast is under the control of the transcription factor TFEB that regulates lysosomal genes, among which is $C L C N 7 .{ }^{62}$ However, the mechanisms by which mutations in CLCN7 regulate ITGB5 expression remains to be determined. CLCN7 mutations act intracellularly. A lack of ClC-7 induces the accumulation of lysosomal storage material, ${ }^{19}$ due to reduced $\mathrm{Cl}^{-}$accumulation in the lysosomes. ${ }^{63}$ Some heterozygous mutations, such as the G215R mutation that we transfected into human osteoclasts, can induce retention of ClC-7 in the endoplasmic reticulum. ${ }^{23,24}$ Some of the mutations retain normal lysosomal targeting, but they induce lower stability or reduce currents. ${ }^{24}$ Although the 
mechanism of the disease may differ depending on the causal mutation, they all impair lysosomal activity, which could impact on the regulation of several genes and thus contribute to impaired osteoclast function.

The objective of osteoporosis treatment is to reduce bone resorption while maintaining or increasing bone formation. It has been proposed that CLCN7 inhibition could be used to treat osteoporosis. ${ }^{64}$ However, increased bone formation has never been demonstrated so far in ADO II. Histomorphometry has evidenced normal bone-forming surfaces in very few patients with ADO II. ${ }^{15}$ They display a continuous increase in bone density over time, ${ }^{8}$ which is compatible with the ongoing bone formation combined with decreased bone resorption. Serum markers of bone formation have not so far been reported in this disease. We observed that none of our patients had reduced bone formation, as could be expected in view of their decreased bone resorption. Moreover, half of them had elevated levels of bone alkaline phosphatases and P1NP. This fits in with the hypothesis that osteoclasts present on bone surfaces can synthesize molecules that directly stimulate bone formation. ${ }^{64,65}$

Recently sphingosine-1-phosphate (S1P), among other factors secreted by osteoclasts, has been shown to stimulate osteoblast differentiation. ${ }^{65}$ More recently, in mice deficient for CTSK, SPHK1 increased expression triggers an higher secretion of S1P by osteoclasts, which has been shown to be responsible for increased bone formation. ${ }^{66}$ We therefore wanted to evaluate sphingosine kinase 1 expression in osteoclasts. It was expressed at the same level in patients and healthy donor osteoclasts. As the number of osteoclasts was higher in the patients, this is compatible with the involvement of sphingosine kinase 1 in the sustained bone formation of these patients.

In conclusion, we have shown that the expression of several genes is modified in osteoclasts from patients with ADO II (ITGB5, WARS, PRF1, and SERPINE2). We were able to demonstrate that integrin $\beta 5$ is overexpressed in the osteoclasts of these patients. Changes in osteoclast motility could explain part of the osteoclast dysfunction of ADO II, and could therefore provide a target for reducing the invalidating clinical symptoms of patients. A knock-in model of ADO II could be used in the future to test this hypothesis.

Supplementary Information accompanies the paper on the Laboratory Investigation website (http://www.laboratoryinvestigation.org)

\section{ACKNOWLEDGMENTS}

This work was performed with funding from the E-rare Grant OSTEOPETR, the SYBIL Grant and the Telethon Grant No. GGP09018. Study design: AEC, AT, and MCDV. Study conduct: AEC and MCDV. Data collection: AEC, ADF, $R O, C B, C C$, and CS. Data analysis: $A E C, R O$, and MCDV. Data interpretation: $A E C, R O$, and MCDV. Drafting manuscript: AEC and MCDV. Revising manuscript content: AEC, ADF, RO, CC, AT, and MCDV. Approving final version of manuscript: $A E C, A D F, R O, C C, A T$, and MCDV. AEC, RO, and MCDV take responsibility for the integrity of the data analysis.

\section{DISCLOSURE/CONFLICT OF INTEREST}

The authors declare no conflict of interest.

1. Del Fattore A, Cappariello A, Teti A. Genetics, pathogenesis and complications of osteopetrosis. Bone 2008;42:19-29.

2. de Vernejoul MC, Kornak U. Heritable sclerosing bone disorders: presentation and new molecular mechanisms. Ann N Y Acad Sci 2010; 1192:269-277.

3. Kornak U, Kasper D, Bosl MR, et al. Loss of the CIC-7 chloride channel leads to osteopetrosis in mice and man. Cell 2001;104:205-215.

4. Chalhoub N, Benachenhou N, Rajapurohitam V, et al. Grey-lethal mutation induces severe malignant autosomal recessive osteopetrosis in mouse and human. Nat Med 2003;9:399-406.

5. Sobacchi C, Frattini A, Guerrini MM, et al. Osteoclast-poor human osteopetrosis due to mutations in the gene encoding RANKL. Nat Genet 2007;39:960-962.

6. Villa A, Guerrini MM, Cassani B, et al. Infantile malignant, autosomal recessive osteopetrosis: the rich and the poor. Calcif Tissue Int 2009; 84:1-12.

7. Bollerslev J, Andersen Jr PE. Radiological, biochemical and hereditary evidence of two types of autosomal dominant osteopetrosis. Bone 1988;9:7-13.

8. Waguespack SG, Hui SL, Dimeglio LA, et al. Autosomal dominant osteopetrosis: clinical severity and natural history of 94 subjects with a chloride channel 7 gene mutation. J Clin Endocrinol Metab 2007;92: 771-778.

9. Benichou OD, Laredo JD, de Vernejoul MC. Type II autosomal dominant osteopetrosis (Albers-Schonberg disease): clinical and radiological manifestations in 42 patients. Bone 2000;26:87-93.

10. Cleiren $\mathrm{E}$, Benichou $\mathrm{O}$, Van Hul E, et al. Albers-Schonberg disease (autosomal dominant osteopetrosis, type II) results from mutations in the CICN7 chloride channel gene. Hum Mol Genet 2001;10:2861-2867.

11. Chu K, Koller DL, Snyder R, et al. Analysis of variation in expression of autosomal dominant osteopetrosis type 2: searching for modifier genes. Bone 2005;37:655-661.

12. Kornak U, Ostertag A, Branger S, et al. Polymorphisms in the CLCN7 gene modulate bone density in postmenopausal women and in patients with autosomal dominant osteopetrosis type II. J Clin Endocrinol Metab 2006;91:995-1000.

13. Campos-Xavier AB, Saraiva JM, Ribeiro LM, et al. Chloride channel 7 (CLCN7) gene mutations in intermediate autosomal recessive osteopetrosis. Hum Genet 2003;112:186-189.

14. Pangrazio $A$, Pusch $M$, Caldana $E$, et al. Molecular and clinical heterogeneity in CLCN7-dependent osteopetrosis: report of 20 novel mutations. Hum Mutat 2010;31:E1071-E1080.

15. Bollerslev J, Marks Jr. SC, Pockwinse S, et al. Ultrastructural investigations of bone resorptive cells in two types of autosomal dominant osteopetrosis. Bone 1993;14:865-869.

16. Estevez R, Jentsch TJ. CLC chloride channels: correlating structure with function. Curr Opin Struct Biol 2002;12:531-539.

17. Weinert $S$, Jabs $S$, Supanchart $C$, et al. Lysosomal pathology and osteopetrosis upon loss of $\mathrm{H}^{+}$-driven lysosomal $\mathrm{Cl}^{-}$accumulation. Science 2010;328:1401-1403.

18. Lange PF, Wartosch $\mathrm{L}$, Jentsch TJ, et al. ClC-7 requires Ostm1 as a betasubunit to support bone resorption and lysosomal function. Nature 2006;440:220-223.

19. Wartosch L, Fuhrmann JC, Schweizer M, et al. Lysosomal degradation of endocytosed proteins depends on the chloride transport protein CIC-7. FASEB J 2009;23:4056-4068.

20. Frattini A, Pangrazio A, Susani $L$, et al. Chloride channel CICN7 mutations are responsible for severe recessive, dominant, and intermediate osteopetrosis. J Bone Miner Res 2003;18:1740-1747.

21. Letizia C, Taranta A, Migliaccio S, et al. Type II benign osteopetrosis (Albers-Schonberg disease) caused by a novel mutation in CLCN7 presenting with unusual clinical manifestations. Calcif Tissue Int 2004; 74:42-46.

22. Wang $\mathrm{C}$, Zhang $\mathrm{H}, \mathrm{He} \mathrm{JW}$, et al. The virulence gene and clinical phenotypes of osteopetrosis in the Chinese population: six novel mutations of the CLCN7 gene in twelve osteopetrosis families. J Bone Miner Metab 2012;30:338-348.

23. Schulz P, Werner J, Stauber T, et al. The G215R mutation in the $\mathrm{Cl}^{-}$/ $\mathrm{H}^{+}$-antiporter CIC-7 found in ADO II osteopetrosis does not abolish 
function but causes a severe trafficking defect. PLoS One 2010; 5:e12585.

24. Leisle L, Ludwig CF, Wagner FA, et al. ClC-7 is a slowly voltage-gated $2 \mathrm{Cl}(-) / 1 \mathrm{H}(+)$-exchanger and requires Ostm 1 for transport activity. EMBO J 2011;30:2140-2152.

25. Henriksen K, Gram J, Hoegh-Andersen P, et al. Osteoclasts from patients with autosomal dominant osteopetrosis type I caused by a T253I mutation in low-density lipoprotein receptor-related protein 5 are normal in vitro, but have decreased resorption capacity in vivo. Am J Pathol 2005;167:1341-1348.

26. Del Fattore A, Peruzzi B, Rucci N, et al. Clinical, genetic, and cellular analysis of 49 osteopetrotic patients: implications for diagnosis and treatment. J Med Genet 2006;43:315-325.

27. Chu K, Snyder R, Econs MJ. Disease status in autosomal dominant osteopetrosis type 2 is determined by osteoclastic properties. J Bone Miner Res 2006;21:1089-1097.

28. Chamoux E, Couture J, Bisson M, et al. The p62 P392L mutation linked to Paget's disease induces activation of human osteoclasts. Mol Endocrinol 2009;23:1668-1680

29. Pruitt KD, Tatusova T, Maglott DR. NCBI reference sequences (RefSeq): a curated non-redundant sequence database of genomes, transcripts and proteins. Nucleic Acids Res 2007;35:D61-D65.

30. Bustin SA, Benes V, Garson JA, et al. The MIQE guidelines: minimum information for publication of quantitative real-time PCR experiments. Clin Chem 2009:55:611-622.

31. Vandesompele J, De Preter K, Pattyn F, et al. Accurate normalization of real-time quantitative RT-PCR data by geometric averaging of multiple internal control genes. Genome Biol 2002;3, RESEARCH0034: 1-12.

32. Taylor A, Rogers MJ, Tosh D, et al. A novel method for efficient generation of transfected human osteoclasts. Calcif Tissue Int 2007:80:132-136.

33. Bollerslev J, Henriksen K, Frost M, et al. Autosomal dominant osteopetrosis revisited: lessons from recent studies. Eur J Endocrinol/Eur Fed Endocr Soc 2013;169:R39-R57.

34. Alatalo SL, Ivaska KK, Waguespack SG, et al. Osteoclast-derived serum tartrate-resistant acid phosphatase $5 \mathrm{~b}$ in Albers-Schonberg disease (type II autosomal dominant osteopetrosis). Clin Chem 2004;50: 883-890.

35. Huang da W, Sherman BT, Lempicki RA. Systematic and integrative analysis of large gene lists using DAVID bioinformatics resources. Nat Protocols 2009;4:44-57.

36. Huang da W, Sherman BT, Lempicki RA. Bioinformatics enrichment tools: paths toward the comprehensive functional analysis of large gene lists. Nucleic Acids Res 2009;37:1-13.

37. Michou L, Chamoux E, Couture J, et al. Gene expression profile in osteoclasts from patients with Paget's disease of bone. Bone 2010; 46:598-603.

38. Nagy ZB, Gergely P, Donath J, et al. Gene expression profiling in Paget's disease of bone: upregulation of interferon signaling pathways in pagetic monocytes and lymphocytes. J Bone Miner Res 2008; 23:253-259.

39. Day IN, Thompson RJ. UCHL1 (PGP 9.5): neuronal biomarker and ubiquitin system protein. Progr Neurobiol 2010;90:327-362.

40. Vestal DJ. The guanylate-binding proteins (GBPs): proinflammatory cytokine-induced members of the dynamin superfamily with unique GTPase activity. J Interferon Cytokine Res 2005;25:435-443.

41. Satoh $\mathrm{T}$, Hemmerlein $\mathrm{B}$, Zschunke $\mathrm{F}$, et al. In situ detection of human monocyte/macrophage serine esterase-1 mRNA expression in human tissues. Pathobiology 1999:67:158-162.

42. Huntington JA. Shape-shifting serpins-advantages of a mobile mechanism. Trends Biochem Sci 2006;31:427-435.

43. Demeo DL, Mariani TJ, Lange C, et al. The SERPINE2 gene is associated with chronic obstructive pulmonary disease. Am J Hum Genet 2006; 78:253-264.

44. Bouton MC, Boulaftali $Y$, Richard B, et al. Emerging role of serpinE2/ protease nexin-1 in hemostasis and vascular biology. Blood 2012;119: 2452-2457.
45. Yang JN, Allan EH, Anderson Gl, et al. Plasminogen activator system in osteoclasts. J Bone Miner Res 1997;12:761-768.

46. Daci $\mathrm{E}$, Udagawa $\mathrm{N}$, Martin $\mathrm{TJ}$, et al. The role of the plasminogen system in bone resorption in vitro. J Bone Miner Res 1999;14: 946-952.

47. Pipkin ME, Rao A, Lichtenheld MG. The transcriptional control of the perforin locus. Immunol Rev 2010;235:55-72.

48. Pipkin ME, Lieberman J. Delivering the kiss of death: progress on understanding how perforin works. Curr Opin Immunol 2007;19: 301-308.

49. Zhou F. Perforin: more than just a pore-forming protein. Int Rev Immunol 2010;29:56-76.

50. Chowdhury D, Lieberman J. Death by a thousand cuts: granzyme pathways of programmed cell death. Annu Rev Immunol 2008;26: 389-420.

51. Kagi D, Ledermann B, Burki K, et al. Cytotoxicity mediated by T cells and natural killer cells is greatly impaired in perforin-deficient mice. Nature 1994:369:31-37.

52. Stepp SE, Dufourcq-Lagelouse R, Le Deist $F$, et al. Perforin gene defects in familial hemophagocytic lymphohistiocytosis. Science 1999;286: 1957-1959.

53. Hersh CP, DeMeo DL, Raby BA, et al. Genetic linkage and association analysis of COPD-related traits on chromosome 8p. COPD 2006;3: 189-194.

54. Chabbi-Achengli $\mathrm{Y}$, Coudert AE, Callebert J, et al. Decreased osteoclastogenesis in serotonin-deficient mice. Proc Natl Acad Sci USA 2012;109:2567-2572.

55. Tolstrup $A B$, Bejder $A$, Fleckner J, et al. Transcriptional regulation of the interferon-gamma-inducible tryptophanyl-tRNA synthetase includes alternative splicing. J Biol Chem 1995;270:397-403.

56. Kasper D, Planells-Cases R, Fuhrmann JC, et al. Loss of the chloride channel CIC-7 leads to lysosomal storage disease and neurodegeneration. EMBO J 2005;24:1079-1091.

57. Inoue M, Namba N, Chappel J, et al. Granulocyte macrophage-colony stimulating factor reciprocally regulates alphav-associated integrins on murine osteoclast precursors. Mol Endocrinol 1998;12:1955-1962.

58. McHugh KP, Hodivala-Dilke $\mathrm{K}$, Zheng MH, et al. Mice lacking beta3 integrins are osteosclerotic because of dysfunctional osteoclasts. J Clin Invest 2000;105:433-440.

59. Lane NE, Yao W, Nakamura MC, et al. Mice lacking the integrin beta5 subunit have accelerated osteoclast maturation and increased activity in the estrogen-deficient state. J Bone Miner Res 2005;20: 58-66.

60. Blair HC, Yaroslavskiy BB, Robinson LJ, et al. Osteopetrosis with microlacunar resorption because of defective integrin organization. Lab Invest 2009;89:1007-1017.

61. Faccio R, Teitelbaum SL, Fujikawa K, et al. Vav3 regulates osteoclast function and bone mass. Nat Med 2005;11:284-290.

62. Lacombe J, Karsenty G, Ferron M. Regulation of lysosome biogenesis and functions in osteoclasts. Cell Cycle 2013;12:2744-2752.

63. Novarino G, Weinert S, Rickheit G, et al. Endosomal chloride-proton exchange rather than chloride conductance is crucial for renal endocytosis. Science 2010;328:1398-1401.

64. Karsdal MA, Henriksen K, Sorensen MG, et al. Acidification of the osteoclastic resorption compartment provides insight into the coupling of bone formation to bone resorption. Am J Pathol 2005; 166:467-476.

65. Pederson L, Ruan $\mathrm{M}$, Westendorf JJ, et al. Regulation of bone formation by osteoclasts involves Wnt/BMP signaling and the chemokine sphingosine-1-phosphate. Proc Natl Acad Sci USA 2008; 105:20764-20769.

66. Lotinun S, Kiviranta R, Matsubara T, et al. Osteoclast-specific cathepsin $\mathrm{K}$ deletion stimulates S1P-dependent bone formation. J Clin Invest 2013;123:666-681.

67. Dutzler R, Campbell EB, Cadene $\mathrm{M}$, et al. X-ray structure of a $\mathrm{CIC}$ chloride channel at 3.0 A reveals the molecular basis of anion selectivity. Nature 2002;415:287-294. 